\title{
Hemorragia digestiva masiva como manifestación de un diverticulo yeyunal
}

\author{
Massive Digestive Bleeding caused by a jejunal diverticulum
}

\author{
Elvira Vaillo Martin ${ }^{1}$, Laura Millán Paredes ${ }^{1}$, Alberto Márquez Rodriguez², \\ Aitor Costales Sánchez ${ }^{3}$, Juan de Diego Gamarra ${ }^{1}$, Andrea Rossetti ${ }^{1}$ \\ 1. Hospital Doctor Jose Molina Orosa de Lanzarote, Servicio de Cirugía General y Digestiva. Arrecife, Las Palmas, España \\ 2. Hospital Doctor Jose Molina Orosa de Lanzarote, Servicio de Gastroenterologia. Arrecife, Las Palmas, España \\ 3. Hospital Doctor Jose Molina Orosa de Lanzarote, Servicio de Radiología. Arrecife, Las Palmas, España
}

\section{RESUMEN}

Los divertículos yeyunales son infrecuentes, su prevalencia oscila entre un $0.3-4.6 \%$ en autopsias y entre un $0.5-2.3 \%$ en los estudios radiológicos. Es una entidad clínica silente en la mayoría de casos, no obstante, entre sus complicaciones se encuentran perforación, sangrado, diverticulitis y obstrucción intestinal. Presentamos el caso de un varón de 76 años con hipertensión arterial y cardiopatía isquémica crónica que acudió al servicio de urgencias por hemorragia digestiva masiva de resolución quirurgica.

Palabras Claves: divertículo, yeyuno, hemorragia digestiva, cirugía.

\section{ABSTRACT}

Jejunal diverticula are rare, with a prevalence rate of $0.3-4.6 \%$ on autopsy studies and $0.5-2.3 \%$ on radiologic studies. It is a clinically silent disease, but complications may include perforation, bleeding, diverticulitis, or intestinal obstruction. We present the case of a 76 yearsold man with arterial hypertension and chronic ischemic cardiopathy; admitted at our emergency department for a massive digestive bleeding with surgical management

Keywords: diverticula, jejunum, gastrointestinal bleeding , surgery

\section{INTRODUCCIÓN}

Los divertículos yeyunales son infrecuentres, su prevalencia en los estudios de autopsias oscila entre un 0.3-4.6\% y entre un 0.5-2.3\% en los estudios radiológicos ${ }^{1}$. Es una entidad clínica silente en la mayoría de casos, no obstante, entre sus complicaciones se encuentran perforación, sangrado, diverticulitis y obstrucción intestinal.

Las guías clínicas actuales recomiendan una vídeo capsula endoscópica, en casos subagudos o crónicos para evaluar el intestino delgado, tras haber excluido el diagnostico mediante endoscopia.

En un estudio la vídeo cápsula ha demostrado mayor capacidad diagnóstica que el AngioTAC, pese a ello, la vídeo cápsula endoscópica falló en un pequeño número de casos que por el contrario si fueron diagnosticados por AngioTAC. Esto sugiere que la combinación de ambos procedimientos podría mejorar el diagnóstico de los pacientes con hemorragias gastrointestinales no identificadas.

\section{CASO CLÍNICO}

El caso corresponde a un hombre de 76 años con antecedentes de hipertensión arterial y cardiopatía isquémica que acude a urgencias por episodio de presíncope asociado a melenas.

En urgencias se realiza un hemograma objetivándose un Hemoglobina de $4 \mathrm{gr} / \mathrm{dl}$. Posterior a la transfusión de hemoderivados y estabilización hemodinámica se procede a la realización de endoscopia alta y baja, sin identificar la etiología del sangrado. Dada la estabilidad del paciente, se procede a realización de angioTAC donde se objetiva sangrado activo a nivel de un probable diverículo yeyunal (Figura 1).

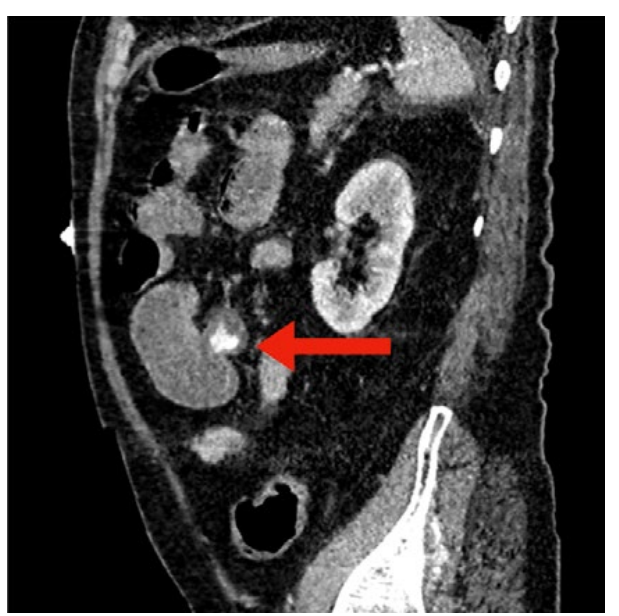

Figura 1. AngioTAC con evidencia de sangrado activo en divertículo. 


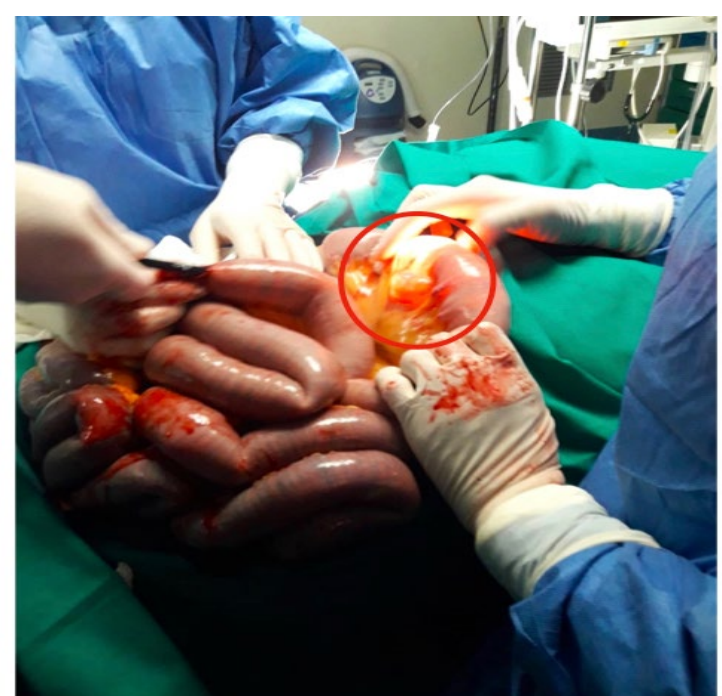

Figura 2. Enteroscopia intraoperatoria con identificación de divertículo yeyunal.

El paciente continúa con anemización y melenas por lo que dada la agudización del caso se opta por laparotomía urgente con enteroscopia intraoperatoria.

Durante el acto quirúgico se procede a la realización de enteroscopia intraoperatoria (Figura 2) que evidencia el divertículo yeyunal con vaso sangrante visible (Figura 3). Se procede a la realización de resección y anastomosis yeyuno-yeyunal.

El paciente presenta una evolución postoperatoria favorable, sin requerir nueva transfusión de hemoderivados siendo dado de alta a los 7 días postoperatorios.

\section{DISCUSIÓN}

En general, el tratamiento del sangrado del divertículo yeyunal está relacionado con la identificación de la etiología del sangrado.

Los pacientes con sintomatología crónica pueden ser manejados de manera conservadora; en caso de persistir sintomatología a pesar del tratamiento se debe considerar la cirugía ${ }^{2}$. Teniendo en cuenta que con la introducción de la enteroscopia de doble balón el sangrado diverticular con vaso visible puede ser controlado con hemoclip ${ }^{5-8}$ en los casos menos complicados.

En caso de sangrado agudo masivo como el presentado, el tratamiento de elección es, previa identificación con enteroscopia intraoperatoria del divertículo sangrante la resección quirúrgica del mismo o del segmento involucrado y anastomosis

\section{REFERENCIAS BIBLIOGRÁFICAS}

1. Yan A, Shindel A, Buxbaum J. Massive obscure bleeding from a jejunal diverticulum (with video). Gastrointest Endosc. 2015;81(5):1289-90.

2. Saperas E, Dot J, Videla S, et al. Capsule endoscopy versus computed tomographic or standard angiography for the diagnosis of obscure gastrointestinal bleeding. Am J Gastroenterol. 2007;102(4):731-7.

3. Hayashi $Y$, Yamamoto $H$, Kita $H$, et al. Non-steroidal anti-inflammatory drug-induced small bowel injuries identified by double-balloon endoscopy. World J Gastroenterol. 2005;11(31):4861-4.

4. Curcio $G$, Ligresti D, Ricotta $C$, et al. Massive bleeding from a jejunal diverticulum reached and treated by underwater single-balloon enteroscopy. Gastrointest Endosc. 2016;84(6):1068-9.

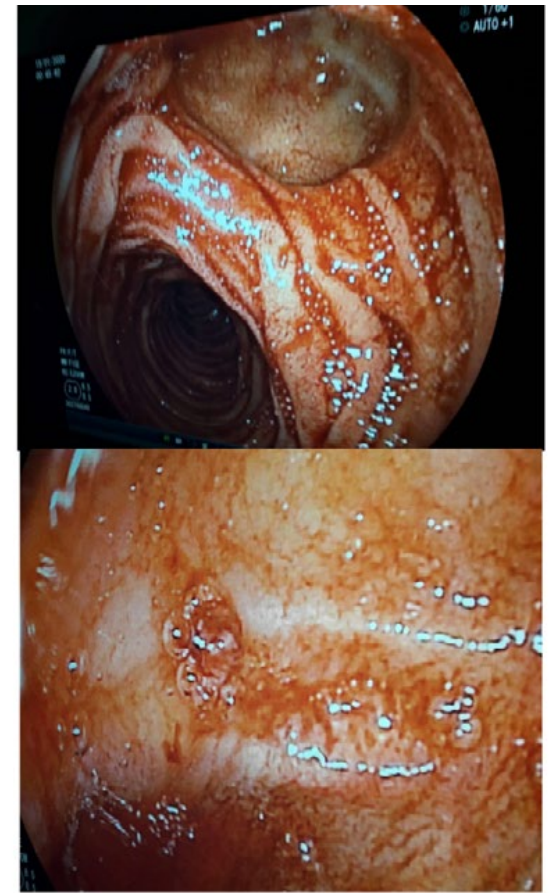

Figura 3. Enteroscopia intraoperatoria con identificación de divertículo y vaso sangrante.

primaria. Los divertículos yeyuno ileales asintomáticos no requierent de tratamiento quirúrgico.

Para concluir queremos remarcar la importancia de un buen diagnóstico con angioTAC así como tratamiento multidisciplinar de cirugía combinada con enteroscopia intraoperatoria.

\section{Responsabilidades éticas}

Protección de personas y animales. Los autores declaran que para esta investigación no se han realizado experimentos en seres humanos ni en animales.

Confidencialidad de los datos. Los autores declaran que han seguido los protocolos de su centro de trabajo sobre la publicación de datos de pacientes.

Derecho a la privacidad y consentimiento informado. Los autores declaran que en este artículo no aparecen datos de pacientes.

Conflicto de intereses. Los autores declaran no tener ningún conflicto de intereses.

5. Fernandes $C$, Pinho $R$, Rodrigues A, et al. Argon plasma coagulation of a bleeding angioectasia in a jejunal diverticulum by single-balloon enteroscopy. Endoscopy. 2015;47(Suppl 1 UCTN):E62.

6. Ikeya T, Ishii N, Shimamura $Y$, et al. Endoscopic band ligation for bleeding lesions in the small bowel. World J Gastrointest Endosc. 2014;6 (10):488-92.

7. Donald JW. Major complications of small bowel diverticula.Ann Surg. 1979;190:183---8.

8. Falidas E, Vlachos K, Mathiolakis S, Archontovasilis F, Villias C.Multiple giant diverticula of the jejunum causing intestinal obs-truction: Report of a case and review of the literature. WorldJ Emerg Surg. 2011;6:1---9. 